\title{
EDITORIAL
}

\section{Illuminating luminal B: QSOX1 as a subtype-specific biomarker}

\author{
Padmalaya Das ${ }^{\dagger}$, Gabrielle M Siegers ${ }^{\dagger}$ and Lynne-Marie Postovit* \\ See related research by Katchman et al., http://breast-cancer-research.com/content/15/2/R28
}

\begin{abstract}
Breast cancer is a complex and heterogeneous disease that affects about one out of every eight women. In the last decade, several advancements have been made that have increased our understanding of breast cancer and have allowed us to more accurately diagnose and treat this disease in a more targeted manner. For example, gene expression profiling enabled the classification of breast cancers into four main subtypes - basal-like, HER2 (human epidermal growth factor receptor 2-positive), luminal $A$ and luminal $B$ - and this classification is used to direct the use of targeted therapies such as tamoxifen or trastuzumab. The luminal subtypes are generally characterized as being estrogen receptor-positive and targetable with anti-hormone therapies. However, whereas luminal A cancers have a good prognosis, luminal $B$ cancers are associated with early relapse following endocrine therapy and a prognosis that is similar to that of the aggressive basal subtype. It is thus imperative that luminal $B$ cancers be better characterized so that therapeutic targets and biomarkers for this disease type can be realized. In the previous issue of Breast Cancer Research, Katchman and colleagues address this need by demonstrating that quiescin sulfydryl oxidase 1 (QSOX1), a secreted enzyme involved in post-translational modifications, is associated with poor prognosis in patients with luminal $\mathrm{B}$ breast cancer. The authors further determined that this protein promotes breast cancer proliferation and invasion. Collectively, these studies suggest that QSOX1 is a predictive biomarker for luminal cancers and that it may be a useful target for elusive luminal $B$ disease.
\end{abstract}

${ }^{\dagger}$ Contributed equally

*Correspondence: Iynne.postovit@schulich.uwo.ca

Department of Anatomy \& Cell Biology and Robarts Research Institute, Western University, 100 Perth Drive, London, ON, Canada N6A 5K8
Targeted therapies improve patient outcome; hence, the quest continues for novel biomarkers and therapeutic targets for breast cancer. One such attempt is described in the previous issue of Breast Cancer Research, in which Katchman and colleagues [1] investigated the role of quiescin sulfydryl oxidase 1 (QSOX1) in luminal B breast cancer. Over the past 13 years, hierarchical clustering of gene expression profiles in patient tumors has been used to divide breast cancer into four main subtypes: basallike, HER2 ${ }^{+}$(human epidermal growth factor receptor 2-positive), luminal A and luminal B [2-5]. The luminal subtypes were originally grouped together on the basis of their luminal epithelial-like gene expression profiles, including the presence of estrogen receptor (ER) [2]. However, they are now recognized as different diseases. Compared with luminal A, luminal B is more aggressive and has higher recurrence rates and shorter survival times after relapse [4]. Moreover, the overall survival of patients with untreated luminal $B$ is similar to that of high-risk patients with basal-like and HER2 ${ }^{+}$breast cancers [6,7]. It is thus imperative that biomarkers and therapeutic targets be developed for luminal B disease. One of the most prominent features of luminal B tumors is their higher grade and proliferative index. For example, proliferation genes such as Cyclin B1 and MKI67 are more highly expressed in luminal B versus luminal A subtypes [6]. However, the immunohistochemical (IHC) detection of Ki67 as a surrogate for proliferation was recently deemed insufficient to clearly distinguish luminal A and B [8]. Thus, definitive delineation of these subtypes is still lacking and the hunt for specific biomarkers continues. Katchman and colleagues [1] suggest that QSOX1 may serve as both a biomarker and therapeutic target for luminal B breast cancer.

QSOX1 is a secreted enzyme that catalyzes the oxidation of thiols to disulfide bonds during protein folding, increasing protein stability [9]. Katchman and colleagues [10] previously showed that QSOX1 increases the invasive potential of a pancreatic cancer cell line by activating matrix metalloproteinase (MMP) 2 and 9. In the present study, the authors employed the Gene expression-based Outcome for Breast cancer Online (GOBO) algorithm, a 
software analysis of Affymetrix data pooled from 11 public data sets [11], to investigate QSOX1 expression in the context of breast cancer. According to GOBO, QSOX1 is highly expressed in luminal A and B breast cancer subtypes but is less expressed in HER2-enriched and basal tumors [1]. Notably, QSOX1 expression was associated with poor relapse-free and overall survival rates in patients with luminal $B$ cancers and with reduced overall survival in patients with luminal A cancers but was not associated with progression when all breast cancers were pooled together. These results suggest that QSOX1 may be a specific prognostic indicator for luminal tumors. IHC staining for QSOX1 on a 153-patient tissue microarray (TMA) showed that while QSOX1 was absent in normal breast tissue, its expression was strongly correlated with higher tumor grade and high Ki-67 expression, both hallmarks of the luminal B subtype [1]. Molecular classifications did not accompany the TMA; thus, attribution of QSOX1 expression to luminal B subtypes can only be implied. Consistent with GOBO and IHC analyses, however, there was increased QSOX1 expression in luminal-like MCF7, MDA-MB-453, ZR 75, and BT474 in comparison with basal-like BT459 and MDA-MB-231 cell lines. Knocking down QSOX1 with short hairpin RNA (shRNA) in MCF7, BT474, and BT459 cells decreased cell growth, suggesting a role for QSOX1 in proliferation. Furthermore, post-translational activation of MMP-9 was implicated in QSOX1-incited cellular invasion. Although this is the first publication correlating QSOX1 expression with the hallmarks of luminal B breast cancer, confirmation of these findings in an animal model as well as subset-stratified patient samples are important next steps.

The findings in this article are similar to those independently reported by Soloviev and colleagues [12] earlier this year. They used expressed sequence tag (EST) and serial analysis of gene expression (SAGE) databases together with real-time polymerase chain reaction of cDNAs from 50 normal and malignant breast tissues to determine that QSOX1 is not expressed in the normal breast and that it is positively correlated with clinical stage. In contrast, a study by Pernodet and colleagues [13] (recently published in this journal) reported that QSOX1 overexpression in breast cancer is associated with good clinical outcome, as QSOX1 levels were inversely correlated to clinical grade and associated with a higher metastasis-free survival. In this study, in vitro experiments using cell lines revealed that QSOX1 decreases cellular proliferation, invasion, and clonogenic potential [13]. In addition, in vivo experiments using mouse xenografts showed that tumor development was enhanced in MDA-MB-231 breast cancer cells in which QSOX1 was knocked down as compared with control cells. It should be noted that molecular classification was not taken into account for the QSOX1 mRNA analyses in the study by Pernodet and colleagues, as was done in the study by Katchman and colleagues [1]. This may have altered Pernodet and colleagues' interpretation of the data regarding the role of QSOX1 in breast cancer progression and metastasis-free survival, and may have concealed subtype-specific trends. Sample size differences may also account for some of the controversial results between these studies: Pernodet and colleagues accessed a cohort of 217 patient samples, whereas the GOBO analysis used by Katchman and colleagues encompassed data from 1,881 patients. Moreover, the IHC analysis conducted by Katchman and colleagues was far more extensive. Hence, their study may have had more power to detect subtype-specific phenomena. The major differences observed regarding the effects of QSOX1 on the behavior of breast cancer cell lines are, however, perplexing. The results published by Katchman and colleagues and Pernodet and colleagues are opposite, with QSOX1 having pro- and anti-tumorigenic properties, respectively. This discordance illustrates the need to further elucidate the role of QSOX1 in breast cancer by using additional models such as primary cells and genetically engineered mice.

Although several agents are currently undergoing clinical trials targeting key signaling pathways in luminal B breast cancer (reviewed in [14]), it is important to continue looking for candidates specifically addressing this and other cancer subtypes offering poor prognoses. While their work clearly warrants further substantiation, preferably in animal models, the focus by Katchman and colleagues on luminal B breast cancer, considered to be the most aggressive form of $\mathrm{ER}^{+}$breast cancer [14], is both timely and laudable.

\section{Abbreviations}

ER, estrogen receptor; GOBO, Gene expression-based Outcome for Breast cancer Online; HER2 ${ }^{+}$, human epidermal growth factor receptor 2-positive; IHC, immunohistochemical; MMP, matrix metalloproteinase; QSOX1, quiescin sulfydryl oxidase 1; TMA, tissue microarray.

\section{Competing interests}

The authors declare that they have no competing interests.

Published: 15 May 2013

\section{References}

1. Katchman BA, Ocal IT, Cunliffe HE, Chang YH, Hostetter G, Watanabe A Lobello J, Lake DF: Expression of quiescin sulfhydryl oxidase 1 is associated with a highly invasive phenotype and correlates with a poor prognosis in luminal B breast cancer. Breast Cancer Res 2013, 15:R28.

2. Perou CM, Sørlie T, Eisen MB, van de Rijn M, Jeffrey SS, Rees CA, Pollack JR, Ross DT, Johnsen H, Akslen LA, Fluge O, Pergamenschikov A, Williams C, Zhu SX, Lønning PE, Børresen-Dale AL, Brown PO, Botstein D: Molecular portraits of human breast tumours. Nature 2000, 406:747-752.

3. Sørlie T, Perou CM, Tibshirani R, Aas T, Geisler S, Johnsen H, Hastie T, Eisen MB, van de Rijn M, Jeffrey SS, Thorsen T, Quist H, Matese JC, Brown PO, Botstein D, Lønning PE, Børresen-Dale AL: Gene expression patterns of breast carcinomas distinguish tumor subclasses with clinical implications. Proc Natl Acad SciU S A 2001, 98:10869-10874. 
4. Sotiriou C, Neo SY, MCShane LM, Korn EL, Long PM, Jazaeri A, Martiat P, Fox SB, Harris AL, Liu ET: Breast cancer classification and prognosis based on gene expression profiles from a population-based study. Proc Natl Acad Sci U S A 2003, 100:10393-10398.

5. Balko JM, Miller TW, Morrison MM, Hutchinson K, Young C, Rinehart C, Sánchez V, Jee D, Polyak K, Prat A, Perou CM, Arteaga CL, Cook RS: The receptor tyrosine kinase ErbB3 maintains the balance between luminal and basal breast epithelium. Proc Nat/ Acad Sci U S A 2012, 109:221-226.

6. Cheang MC, Chia SK, Voduc D, Gao D, Leung S, Snider J, Watson M, Davies S, Bernard PS, Parker JS, Perou CM, Ellis MJ, Nielsen TO: Ki67 index, HER2 status, and prognosis of patients with luminal B breast cancer. J Nat/ Cancer Inst 2009, 101:736-750.

7. Hu Z, Fan C, Oh DS, Marron JS, He X, Qaqish BF, Livasy C, Carey LA, Reynolds E, Dressler L, Nobel A, Parker J, Ewend MG, Sawyer LR, Wu J, Liu Y, Nanda R, Tretiakova M, Ruiz Orrico A, Dreher D, Palazzo JP, Perreard L, Nelson E, Mone M, Hansen H, Mullins M, Quackenbush JF, Ellis MJ, Olopade Ol, Bernard PS, Perou CM: The molecular portraits of breast tumors are conserved across microarray platforms. BMC Genomics 2006, 7:96

8. Guiu S, Michiels S, André F, Cortes J, Denkert C, Di Leo A, Hennessy BT, Sorlie T, Sotiriou C, Turner N, Van de Vijver M, Viale G, Loi S, Reis-Filho JS: Molecular subclasses of breast cancer: how do we define them? The IMPAKT 2012 Working Group Statement. Ann Oncol 2012, 23:2997-3006.

9. Thorpe C, Hoober KL, Raje S, Glynn NM, Burnside J, Turi GK, Coppock DL: Sulfhydryl oxidases: emerging catalysts of protein disulfide bond formation in eukaryotes. Arch Biochem Biophys 2002, 405:1-12.
10. Katchman BA, Antwi K, Hostetter G, Demeure MJ, Watanabe A, Decker GA, Miller LJ, Von Hoff DD, Lake DF: Quiescin sulfhydryl oxidase 1 promotes invasion of pancreatic tumor cells mediated by matrix metalloproteinases. Mol Cancer Res 2011, 9:1621-1631.

11. Ringner M, Fredlund E, Hakkinen J, Borg A, Staaf J: GOBO: gene expressionbased outcome for breast cancer online. PLoS one 2011, 6:e17911.

12. Soloviev M, Esteves MP, Amiri F, Crompton MR, Rider CC: Elevated transcription of the gene QSOX1 encoding quiescin Q6 sulfhydryl oxidase 1 in breast cancer. PLoS one 2013, 8:e57327.

13. Pernodet N, Hermetet F, Adami P, Vejux A, Descotes F, Borg C, Adams M, Pallandre JR, Viennet G, Esnard F, Jouvenot M, Despouy G: High expression of QSOX1 reduces tumorogenesis, and is associated with a better outcome for breast cancer patients. Breast Cancer Res 2012, 14:R136.

14. Tran B, Bedard PL: Luminal-B breast cancer and novel therapeutic targets. Breast Cancer Res 2011, 13:221.

doi: $10.1186 /$ bcr3417

Cite this article as: Das $P$, et al:: Illuminating luminal B: QSOX1 as a subtypespecific biomarker. Breast Cancer Research 2013, 15:104. 